Article

\title{
On Infinitely Many Rational Approximants to $\zeta(3)$
}

\author{
Jorge Arvesú ${ }^{1, *}$ and Anier Soria-Lorente ${ }^{2}$ (D) \\ 1 Department of Mathematics, Universidad Carlos III de Madrid, Avda de la Universidad, 30, \\ 28911 Leganés, Spain \\ 2 Technology Department, University of Granma, Bayamo 85100, Cuba; asorial@udg.co.cu \\ * Correspondence: jarvesu@math.uc3m.es
}

Received: 3 October 2019; Accepted: 23 November 2019; Published: 3 December 2019

\begin{abstract}
A set of second order holonomic difference equations was deduced from a set of simultaneous rational approximation problems. Some orthogonal forms involved in the approximation were used to compute the Casorati determinants for its linearly independent solutions. These solutions constitute the numerator and denominator sequences of rational approximants to $\zeta(3)$. A correspondence from the set of parameters involved in the holonomic difference equation to the set of holonomic bi-sequences formed by these numerators and denominators appears. Infinitely many rational approximants can be generated.
\end{abstract}

Keywords: holonomic difference equation; integer sequences; irrationality; multiple orthogonal polynomials; orthogonal forms; recurrence relation; simultaneous rational approximation

\section{Introduction}

Apéry's proof [1] of the irrationality of the number $\zeta(3)$ (where $\zeta(s)=\sum_{n=1}^{\infty} n^{-s}$, $\operatorname{Re} s>1$ ) is based on the second order difference equation (three-term recurrence relation)

$$
\begin{aligned}
& \alpha_{n} y_{n+1}+\beta_{n} y_{n}+\gamma_{n} y_{n-1}=0, \quad n=1,2, \ldots \\
& \alpha_{n}=(n+1)^{3}, \quad \beta_{n}=-(2 n+1)\left(17 n^{2}+17 n+5\right), \quad \gamma_{n}=n^{3}
\end{aligned}
$$

where the two independent solutions, namely, the sequence of integers $\left(q_{n}\right)_{n \geq 0}$ defined by the initial conditions $q_{0}=1$ and $q_{1}=5$, (more specifically $q_{n}=\sum_{k=0}^{n}\left(\begin{array}{c}n+k \\ k\end{array}\right)^{2}\left(\begin{array}{c}n \\ k\end{array}\right)^{2}[2,3]$ ) and the sequence of rationals $\left(p_{n}\right)_{n \geq 0}$ determined by the initial conditions $p_{0}=0, p_{1}=6$, form rational approximants $\left(p_{n} / q_{n}\right)_{n \geq 0}$ to the number $\zeta(3)$. This sequence of approximants can be expressed as the sequence of convergents of the corresponding continued fraction. Such a sequence shows the irrationality of $\zeta(3)$. Indeed, the inclusions $q_{n}, p_{n} l_{n}^{3} \in \mathbb{Z}$, where $l_{n}$ denotes the least common multiple of $\{1,2, \ldots, n\}$, together with the prime number theorem, $l_{n}=O\left(e^{(1+\epsilon) n}\right)$ for any $\epsilon>0$, and Poincaré's theorem [4-6] yield for the error-term sequence $r_{n}=q_{n} \zeta(3)-p_{n}$, the estimate

$$
\limsup _{n \rightarrow \infty} \sqrt[n]{l_{n}^{3}\left|r_{n}\right|} \leq e^{3}(\sqrt{2}-1)^{4}<1
$$

In [7] the proof is addressed from the perspective of Hermite-Padé approximation problem involving multiple orthogonal polynomials [8-10], but that proof was not connected with Equation (1). A natural question arises: Do the coefficients in (1) depend on other integers as well as $n$ in order that the derived sequences $p_{n}$ and $q_{n}$ guarantee the irrationality of $\zeta(3)$ ? Following this perspective, the primary goal of this paper was to obtain a second order holonomic difference equation as an alternative to the Equation (1) by using rational approximation techniques in the context of Hermite-Padé approximation. In this paper we use the term "holonomic" to indicate that the coefficients in the 
linear difference equation depend on other parameters as well as $n$ (see $[11,12]$ for this notion in the context of differential equations involving some Krall-type and Sobolev-type orthogonal polynomials). The holonomic character of Equation (36) leads to infinitely many sequences of rational approximants to $\zeta(3)$. This is because the holonomic sequences that are produced depend on up to three integer parameters (almost freely selected). Usually, the deduction of the Equation (1) makes use of the algorithm of creative telescoping due to Gosper and Zeilberger [13]. However, the use of this technique is combinatorially more complicated when dealing with holonomic sequences that depend on several parameters.

Our goal was to find and solve a set of second order holonomic difference equations (see (36))

$$
\alpha_{n, \Omega} y_{n+1}^{(i, j)}+\beta_{n, \Omega} y_{n}^{(i, j)}+\gamma_{n, \Omega} y_{n-1}^{(i, j)}=0, \quad n=1,2, \ldots, \quad 1 \leq i \leq 4, \quad 1 \leq j \leq 3,
$$

with initial conditions $q_{0}^{(i, j)}(\Omega), q_{1}^{(i, j)}(\Omega) \in \mathbb{Q}$ and $p_{0}^{(i, j)}(\Omega), p_{1}^{(i, j)}(\Omega) \in \mathbb{Q}$, respectively. The expressions for the coefficients $\alpha_{n, \Omega}, \beta_{n, \Omega}$, and $\gamma_{n, \Omega}$ are given in Section 3. $\Omega \subset \mathbb{Z}^{3}$ represents a set of integer parameters involved in the polynomial solutions of the approximation problems (5) and (6). Let $\Omega=$ $\Omega_{i, j}$, where

$$
\begin{aligned}
& \Omega_{i, 1}=\rho, \quad \rho \in \mathbb{N} \backslash\{1\}, \\
& \Omega_{i, 2}=\vartheta, \quad \vartheta \in \mathbb{Z} \backslash\{-1,0,1\}, \\
& \Omega_{i, 3}=(v, \chi, \psi), \quad v, \chi \in \mathbb{N}, \quad \chi \geq v, \quad \psi \in \mathbb{N} \cup\{0\},
\end{aligned}
$$

and $\mathbb{N}, \mathbb{Z}$, and $\mathbb{Q}$ denote the sets of all positive integers, integer numbers, and rational numbers, respectively.

For a given integer or a combination of given integers as in (3) we found the corresponding holonomic solutions $\left(p_{n}\right)_{n \geq 0}$ and $\left(q_{n}\right)_{n \geq 0}$, which form the numerator and denominator sequences of the rational approximants to $\zeta(3)$. Indeed, we give a correspondence from the set $\Omega$ to the set of bi-sequences $\left(q_{n}, p_{n}\right)$ that are solutions of the holonomic three-term recurrence relations (2) with variable coefficients depending on integer parameters.

The paper is structured as follows. In Section 2 we introduce Hermite-Padé approximation problems near infinity with polynomial solutions. These solutions depend on integer parameters $\Omega$ that satisfy a set of conditions. In Section 3 we compute the Casorati determinants for sequences of numerators and denominators of the rational approximants to $\zeta(3)$. Different series representations of the number $\zeta(3)$ are derived. Moreover, from the Casorati determinant we deduce the second order holonomic difference equation satisfied by the sequences of both numerators and denominators of the rational approximants. In Section 4 we asymptotically estimate the error-term sequence of our approximations by using steepest descent method. Then, we verify that the generated rational approximants (in Section 2.1) and reprove the irrationality of $\zeta(3)$. In Section 5 several comparisons (computations) involving rational approximants and Apéry's approximants are given. We make concluding remarks in Section 6.

\section{Hermite-Padé Approximation}

We derived a set of rational approximants classified in twelve types $p_{n}^{(i, j)} / q_{n}^{(i, j)}(1 \leq i \leq 4,1 \leq$ $j \leq 3)$ depending on certain parameters, which can be used to generate infinitely many rational approximants to $\zeta(3)$. For that purpose, we formulated and solved the following problem:

For the system of polylogarithms $\mathrm{Li}_{s}\left(z^{-1}\right)=\sum_{j=1}^{\infty} z^{-j} / j^{s}, s=1,2,3$, find the polynomials

$$
A_{n}^{(i, j)}(z)=\sum_{k=0}^{n} a_{k, n}^{(i, j)} z^{k}, \quad B_{n-\delta_{i, 3}}^{(i, j)}(z)=\sum_{k=0}^{n-\delta_{i, 3}} b_{k, n-\delta_{i, 3}}^{(i, j)} z^{k}, \quad\left(\delta_{i, j} \text { is the Kronecker delta function }\right)
$$


$C_{n}^{(i, j)}(z)$, and $D_{n}^{(i, j)}(z)$ such that the following interpolation conditions at infinity

$$
\begin{aligned}
& A_{n}^{(i, j)}(z) \operatorname{Li}_{1}\left(z^{-1}\right)+B_{n-\delta_{i, 3}}^{(i, j)}(z) \operatorname{Li}_{2}\left(z^{-1}\right)+C_{n}^{(i, j)}(z)=\mathcal{O}\left(z^{-n-\delta_{i, 1}}\right), \\
& A_{n}^{(i, j)}(z) \operatorname{Li}_{2}\left(z^{-1}\right)+2 B_{n-\delta_{i, 3}}^{(i, j)}(z) \operatorname{Li}_{3}\left(z^{-1}\right)+D_{n}^{(i, j)}(z)=\mathcal{O}\left(z^{-n}\right), \quad i=1,2,3,4, \quad j=1,2,3,
\end{aligned}
$$

hold, provided the following extra conditions involving the coefficients of polynomials (4) are fulfilled

$$
\begin{aligned}
A_{n}^{(i, j)}(1) & =0, \\
\int_{0}^{1} F_{n}^{(i, j)}(x) x^{\alpha} d x=0, \quad \delta_{i, 2} \int_{0}^{1} G_{n}^{(i, j)}(x) x^{\alpha} d x & =0,
\end{aligned}
$$

where

$$
F_{n}^{(i, j)}(x)=A_{n}^{(i, j)}(x)+B_{n-\delta_{i, 3}}^{(i, j)}(x) \log x, \quad G_{n}^{(i, j)}(x)=F_{n}^{(i, j)}(x) \log x
$$

and

$$
\begin{aligned}
\alpha & =\left\{t \in \mathbb{Q}: \theta^{(i, j)}(t)=0\right\} \\
\theta^{(i, j)}(t) & :=\frac{(t+n+1)^{\delta_{i, 3}}\left[\delta_{1, j}(t+n+\rho)+\delta_{2, j}(t+\vartheta n+1)+\delta_{3, j}(v t-\chi n-\psi)\right]^{\delta_{i, 2}+1}}{(t-n+1)^{2-\delta_{i, 1}}}
\end{aligned}
$$

with $\rho \in \mathbb{N} \backslash\{1\}, \vartheta \in \mathbb{Z} \backslash\{-1,0,1\}, v, \chi \in \mathbb{N}(\chi \geq v), \psi \in \mathbb{N} \cup\{0\}$.

Condition (7) implies the following relation

$$
2 \zeta(3) \sum_{k=0}^{n-\delta_{i, 3}} b_{k, n-\delta_{i, 3}}^{(i, j)}+D_{n}^{(i, j)}(1)=r_{n}^{(i, j)}=\text { error-term sequence, }
$$

since $\operatorname{Li}_{s}(1)=\zeta(s), \operatorname{Re} s>1$, while conditions (8)-(10) introduce certain dependence of parameters in the coefficients of polynomials (4), and consequently, in the coefficients of polynomials $C_{n}^{(i, j)}(z)$ and $D_{n}^{(i, j)}(z)$, respectively (see expressions (17) and (18) below).

For each pair $(i, j)$, the interpolation problems (5) and (6), together with (7) and (8), leads to a linear system of equations for the unknown coefficients of $A_{n}^{(i, j)}(z), B_{n-\delta_{i, 3}}^{(i, j)}(z), C_{n}^{(i, j)}(z)$, and $D_{n}^{(i, j)}(z)$, which are uniquely determined (see [10]).

Focusing on the polynomial solutions of (5) and (6), we show that such solutions can be derived from the orthogonality relations (12) with conditions (7) and (8), instead of solving the aforementioned linear system of equations. Let $A_{n}^{(i, j)}(x)$ and $B_{n-\delta_{i, 3}}^{(i, j)}(x)$ be two polynomials, such that $F_{n}^{(i, j)}(x)$ and $G_{n}^{(i, j)}(x)$ are as in (9). Let

$$
\begin{gathered}
\int_{0}^{1} F_{n}^{(i, j)}(x) x^{k} d x=0, \quad k=0, \ldots, n-2+\delta_{i, 1} \\
\int_{0}^{1} G_{n}^{(i, j)}(x) x^{k} d x=0, \quad k=0, \ldots, n-2,
\end{gathered}
$$

be subject to the conditions (7) and (8). These relations lead to a linear system of equations for the unknown coefficients of $A_{n}^{(i, j)}(x)$ and $B_{n-\delta_{i, 3}}^{(i, j)}(x)$, respectively. As a consequence of (12) we have

$$
\begin{aligned}
& \int_{0}^{1} p(x) \frac{F_{n}^{(i, j)}(x)}{z-x} d x=p(z) \int_{0}^{1} \frac{F_{n}^{(i, j)}(x)}{z-x} d x, \\
& \int_{0}^{1} q(x) \frac{G_{n}^{(i, j)}(x)}{z-x} d x=q(z) \int_{0}^{1} \frac{G_{n}^{(i, j)}(x)}{z-x} d x,
\end{aligned}
$$


where $p(z)$ and $q(z)$ are arbitrary polynomials of degree at most $n+\delta_{i, 1}-1$ and $n-1$, respectively.

Denoting

$$
r_{n, 1}^{(i, j)}(z)=\int_{0}^{1} \frac{F_{n}^{(i, j)}(x)}{z-x} d x, \quad r_{n, 2}^{(i, j)}(z)=\int_{0}^{1} \frac{G_{n}^{(i, j)}(x)}{z-x} d x,
$$

Equation (13) implies that $r_{n, 1}^{(i, j)}(z)=\mathcal{O}\left(z^{-n-\delta_{i, 1}}\right)$ and $r_{n, 2}^{(i, j)}(z)=\mathcal{O}\left(z^{-n}\right)$. Now, setting $p(z)=q(z)=1$ in Equations (13) and using (9), one obtains

$$
\begin{aligned}
\int_{0}^{1} \frac{F_{n}^{(i, j)}(x)}{z-x} d x & =\int_{0}^{1} \frac{A_{n}^{(i, j)}(x)+B_{n-\delta_{i, 3}}^{(i, j)}(x) \log x}{z-x} d x \\
& =A_{n}^{(i, j)}(z) \operatorname{Li}_{1}\left(z^{-1}\right)+B_{n-\delta_{i, 3}}^{(i, j)}(z) \operatorname{Li}_{2}\left(z^{-1}\right)-C_{n}^{(i, j)}(z) \\
\int_{0}^{1} \frac{G_{n}^{(i, j)}(x)}{z-x} d x & =\int_{0}^{1} \frac{A_{n}^{(i, j)}(x)+B_{n-\delta_{i, 3}}^{(i, j)}(x) \log x}{z-x} \log x d x \\
& =A_{n}^{(i, j)}(z) \operatorname{Li}_{2}\left(z^{-1}\right)+2 B_{n-\delta_{i, 3}}^{(i, j)}(z) \operatorname{Li}_{3}\left(z^{-1}\right)-D_{n}^{(i, j)}(z)
\end{aligned}
$$

where

$$
\begin{aligned}
\mathrm{Li}_{k}\left(z^{-1}\right) & =\frac{1}{(k-1) !} \int_{0}^{1} \frac{\log ^{k-1} x}{z-x} d x, \quad k=1,2,3, \\
C_{n}^{(i, j)}(z) & =\int_{0}^{1} \frac{A_{n}^{(i, j)}(z)+B_{n-\delta_{i, 3}(i, j)}(z) \log x-F_{n}^{(i, j)}(x)}{z-x} d x, \\
D_{n}^{(i, j)}(z) & =\int_{0}^{1} \frac{\left(A_{n}^{(i, j)}(z)+B_{n-\delta_{i, 3}}^{(i, j)}(z) \log x\right) \log x-G_{n}^{(i, j)}(x)}{z-x} d x .
\end{aligned}
$$

Therefore, the above system of functions (16) and polynomials (4), (17), and (18) represent the simultaneous rational approximation problem near infinity (5) and (6). Notice that the orthogonality conditions (12) along with (7) and (8) are equivalent to the interpolation conditions at infinity, (5) and (6). The solution of this problem depends only on the coefficients of the polynomials $A_{n}^{(i, j)}(z)$ and $B_{n-\delta_{i, 3}}^{(i, j)}(z)$, since the coefficients for $z^{-v}\left(1 \leq v \leq n-1+\delta_{i, 1}\right)$ in the Laurent series expansion of $A_{n}^{(i, j)}(z) \operatorname{Li}_{1}\left(z^{-1}\right)+B_{n-\delta_{i, 3}}^{(i, j)}(z) \operatorname{Li}_{2}\left(z^{-1}\right)$ and for $z^{-v}(1 \leq v \leq n-1)$ in the series expansion of $A_{n}^{(i, j)}(z) \operatorname{Li}_{2}\left(z^{-1}\right)+2 B_{n-\delta_{i, 3}}^{(i, j)}(z) \operatorname{Li}_{3}\left(z^{-1}\right)$ vanish, while the coefficients for $z^{v}(0 \leq v \leq n)$ coincide with the corresponding coefficients of $C_{n}^{(i, j)}(z)$ and $D_{n}^{(i, j)}(z)$, respectively.

\subsection{Explicit Expressions for Coefficients of the Vector Polynomial}

We determined the common vector denominator $\left(A_{n}^{(i, j)}(z), B_{n-\delta_{i, 3}}^{(i, j)}(z)\right)$ for the vector type II approximation problems (14) and (15). Define

$$
R_{1, n}^{(i, j)}(t)=\int_{0}^{1} F_{n}^{(i, j)}(x) x^{t} d x, \quad R_{2, n}^{(i, j)}(t)=\int_{0}^{1} G_{n}^{(i, j)}(x) x^{t} d x, \quad(t>-1) .
$$

Thus, we have the following sequences of rational functions

$$
R_{1, n}^{(i, j)}(t)=\sum_{k=0}^{n} \frac{a_{k, n}^{(i, j)}}{t+k+1}-\sum_{k=0}^{n-\delta_{i, 3}} \frac{b_{k, n-\delta_{i, 3}}^{(i, j)}}{(t+k+1)^{2}}, \quad n-\delta_{i, 3} \geq 0,
$$

and

$$
R_{2, n}^{(i, j)}(t)=2 R_{1, n}^{(i, j)}(t)\left[\sum_{k=0}^{n-2} \frac{1}{t-k}-\sum_{k=1}^{n-\delta_{i, 3}+1} \frac{1}{t+k}+\varphi^{(i, j)}(t)\right]
$$


where

$$
\varphi^{(i, j)}(t)=\frac{d}{d t} \log \theta^{(i, j)}(t), \quad \text { and } \quad R_{2, n}^{(i, j)}(t)=\frac{d}{d t} R_{1, n}^{(i, j)}(t) .
$$

The expressions (20) and (21) represent the analytic continuation of the two functions given in (19), respectively.

From the orthogonality conditions (13), as well as from (7) and (8), it follows that

$$
R_{1, n}^{(i, j)}(t)=R_{1, n}(t) \theta^{(i, j)}(t), \quad \text { where } \quad R_{1, n}(t)=\frac{(-t)_{n}^{2}}{(t+1)_{n+1}^{2}}, \quad n \in \mathbb{N}
$$

and $(t)_{n}=t(t+1) \cdots(t+n-1),(t)_{0}=1$, is the Pochhammer symbol.

Hence, the coefficients in (20) are as follows

$$
\begin{aligned}
b_{k, n-\delta_{i, 3}}^{(i, j)} & =-\lim _{t \rightarrow-(k+1)}(t+k+1)^{2} R_{1, n}^{(i, j)}(t), \quad k=0, \ldots, n-\delta_{i, 3} \\
& =-\left(\begin{array}{c}
n+k \\
k
\end{array}\right)^{2}\left(\begin{array}{l}
n \\
k
\end{array}\right)^{2} \theta^{(i, j)}(-k-1), \quad b_{0, n-\delta_{i, 3}}^{(i, j)}=-\theta^{(i, j)}(-1),
\end{aligned}
$$

and

$$
\begin{aligned}
a_{k, n}^{(i, j)} & =\operatorname{Res}_{t=-k-1} R_{1, n}^{(i, j)}(t), \quad k=0, \ldots, n-\delta_{i, 3}, \\
& =2 b_{k, n-\delta_{i, 3}}^{(i, j)}\left[H_{n+k-1}-2 H_{k}+H_{n-k-\delta_{i, 3}}-\varphi^{(i, j)}(-k-1)\right],
\end{aligned}
$$

where $H_{k}^{(r)}$ denotes the Harmonic Number $k$ of order $r\left(H_{k}^{(1)}=H_{k}\right.$ and $\left.H_{0}=0\right)$. In addition, the following relation holds

$$
a_{n, n}^{(3, j)}=\lim _{t \rightarrow-(n+1)}(t+n+1) R_{1, n}^{(3, j)}(t)=\frac{(-1)^{\delta_{3, j}}}{n^{2-\delta_{2, j}}}\left(\begin{array}{c}
2 n-1 \\
n-1
\end{array}\right)^{2} \sigma_{3, j}, \quad j=1,2,3,
$$

where $\sigma_{3,1}=\rho-1, \sigma_{3,2}=\vartheta-1, \sigma_{3,3}=v(n+1)+\chi n+\psi$. Observe that if $l_{n}$ denotes the least common multiple of $\{1,2, \ldots, n\}$, and $k=0, \ldots, n-\delta_{i, 3}, m=0, \ldots, n$, the following inclusions

$$
n^{\omega_{i, j}} b_{k, n-\delta_{i, 3^{\prime}}}^{(i, j)} n^{\omega_{i, j}} l_{n} a_{m, n}^{(i, j)} \in \mathbb{Z}, \quad \text { where } \quad\left(\omega_{i, j}\right)_{4,3}=\left(\begin{array}{lll}
1 & 0 & 1 \\
2 & 0 & 2 \\
1 & 0 & 1 \\
2 & 1 & 2
\end{array}\right)
$$

hold.

We now derive an explicit expression for the sequences involved in (11). From expressions (11), (18), and (23)-(25) one gets

$$
q_{n}^{(i, j)} \zeta(3)-p_{n}^{(i, j)}=r_{n}^{(i, j)}, \quad n-\delta_{i, 3} \geq 0,
$$

where

$$
q_{n}^{(i, j)}=2 \sum_{k=0}^{n-\delta_{i, 3}} b_{k, n-\delta_{i, 3^{\prime}}}^{(i, j)} \quad p_{n}^{(i, j)}=D_{n}^{(i, j)}(1)=2 \sum_{k=1}^{n-\delta_{i, 3}} b_{k, n-\delta_{i, 3}}^{(i, j)} H_{k}^{(3)}-\sum_{k=1}^{n} a_{k, n}^{(i, j)} H_{k}^{(2)}, \quad\left(p_{0}^{(i, j)}=0\right) .
$$

Finally, since $R_{1, n}^{(i, j)}(t)=\mathcal{O}\left(t^{-2-\delta_{i, 3}}\right)$ as $t \rightarrow \infty$, we have

$$
\sum_{k=0}^{n} a_{k, n}^{(i, j)}=\sum_{k=0}^{n} \operatorname{Res}_{t=-k-1} R_{1, n}^{(i, j)}(t)=-\operatorname{Res}_{t=\infty} R_{1, n}^{(i, j)}(t)=0,
$$


which is in accordance with (7).

\section{Second Order Holonomic Difference Equation}

We begin by obtaining an explicit expression for the Casorati determinant involving the above functions (19)-(21) and sequences (23)-(27); i.e.

$$
\begin{aligned}
& W\left(q_{n}^{(i, j)}, r_{n}^{(i, j)}\right)=\operatorname{det}\left(\begin{array}{ll}
q_{n}^{(i, j)} & r_{n}^{(i, j)} \\
q_{n+1}^{(i, j)} & r_{n+1}^{(i, j)}
\end{array}\right)=-W\left(q_{n}^{(i, j)}, p_{n}^{(i, j)}\right), \quad n \geq 1, \\
& W\left(q_{0}^{(i, j)}, r_{0}^{(i, j)}\right)=-q_{0}^{(i, j)} p_{1}^{(i, j)} .
\end{aligned}
$$

Lemma 1. The following relation

$$
\begin{aligned}
W\left(q_{n}^{(i, j)}, p_{n}^{(i, j)}\right) & =2\left[a_{n+1, n+1}^{(i, j)} R_{1, n}^{(i, j)}(n)+b_{n-\delta_{i, 3}+1, n-\delta_{i, 3}+1}^{(i, j)}\left(R_{2, n}^{(i, j)}(n)+R_{2, n}^{(i, j)}(n-1)\right)\right. \\
& \left.+\quad b_{n-\delta_{i, 3}, n-\delta_{i, 3}+1}^{(i, j)} R_{2, n}^{(i, j)}(n-1)\right], \quad n \geq 1
\end{aligned}
$$

holds.

Proof. Consider the integral

$$
I_{n, \Omega}=2 \int_{0}^{1} \frac{F_{n}^{(i, j)}(x) F_{n+1}^{(i, j)}(x)}{1-x} d x, \quad n \geq 1 .
$$

Let $\Omega$ represent the generic dependence of the parameters $\rho, \vartheta, v, \chi$, and $\psi$ defined in (3). Using formulas (13) and the fact that $A_{n}^{(i, j)}(1)=0$, one gets

$$
I_{n, \Omega}=2 B_{n-\delta_{i, 3}}^{(i, j)}(1) \int_{0}^{1} \frac{G_{n+1}^{(i, j)}(x)}{1-x} d x=q_{n}^{(i, j)} r_{n+1}^{(i, j)}
$$

On the other hand,

$$
\frac{F_{n}^{(i, j)}(x) F_{n+1}^{(i, j)}(x)}{1-x}=B_{n-\delta_{i, 3}+1}^{(i, j)}(1) \frac{G_{n}^{(i, j)}(x)}{1-x}-F_{n}^{(i, j)}(x)\left(\tilde{A}_{n}^{(i, j)}(x)+\tilde{B}_{n-\delta_{i, 3}}^{(i, j)}(x) \log x\right),
$$

where

$$
\tilde{A}_{n}^{(i, j)}(x)=\frac{A_{n+1}^{(i, j)}(1)-A_{n+1}^{(i, j)}(x)}{1-x}, \quad \tilde{B}_{n-\delta_{i, 3}}^{(i, j)}(x)=\frac{B_{n-\delta_{i, 3}+1}^{(i, j)}(1)-B_{n-\delta_{i, 3}+1}^{(i, j)}(x)}{1-x} .
$$

Hence,

$$
\begin{aligned}
I_{n, \Omega} & =2 \int_{0}^{1} \frac{F_{n}^{(i, j)}(x) F_{n+1}^{(i, j)}(x)}{1-x} d x=q_{n+1}^{(i, j)} r_{n}^{(i, j)}-2\left[a_{n+1, n+1}^{(i, j)} R_{1, n}^{(i, j)}(n)\right. \\
& \left.+\quad b_{n-\delta_{i, 3}+1, n-\delta_{i, 3}+1}^{(i, j)}\left(R_{2, n}^{(i, j)}(n)+R_{2, n}^{(i, j)}(n-1)\right)+b_{n-\delta_{i, 3}, n-\delta_{i, 3}+1}^{(i, j)} R_{2, n}^{(i, j)}(n-1)\right] .
\end{aligned}
$$

Equating (30) and (31), one obtains (29).

The first-order linear inhomogeneous difference equation with variable coefficients involved in the Casorati determinant (29) can be written as follows

$$
h_{n+1}=f_{n} h_{n}+g_{n}, \quad f_{n} \neq 0, \quad n \geq 1,
$$


where $h_{n}=r_{n}^{(i, j)}, f_{n}=q_{n+1}^{(i, j)} / q_{n}^{(i, j)}, g_{n}=W\left(q_{n}^{(i, j)}, r_{n}^{(i, j)}\right) / q_{n}^{(i, j)}$. Observe that its solution is given by

$$
h_{n}=\left(\prod_{m=0}^{n-1} f_{m}\right)\left(A_{0}+\sum_{m=0}^{n-1} \frac{g_{m}}{\prod_{k=0}^{m} f_{k}}\right), \quad A_{0}=\zeta(3) q_{0}^{(i, j)} .
$$

Hence, from (32) we have

$$
r_{n}^{(i, j)}=\frac{q_{n}^{(i, j)}}{q_{0}^{(i, j)}}\left(A_{0}-q_{0}^{(i, j)} \sum_{m=0}^{n-1} \frac{W\left(q_{m}^{(i, j)}, p_{m}^{(i, j)}\right)}{q_{m+1}^{(i, j)} q_{m}^{(i, j)}}\right),
$$

and

$$
p_{n}^{(i, j)}=q_{n}^{(i, j)} \sum_{m=0}^{n-1} \frac{W\left(q_{m}^{(i, j)}, p_{m}^{(i, j)}\right)}{q_{m+1}^{(i, j)} q_{m}^{(i, j)}}, \quad n \geq 1 .
$$

Expression (34) gives a different representation for the sequences of numerators (28) of the rational approximants to the number $\zeta(3)$ provided one is able to explicitly compute (29). Moreover, both from Theorem 2 (see Section 4) and from Theorem 1 below $\lim _{n \rightarrow \infty} r_{n}^{(i, j)} / q_{n}^{(i, j)}=0$. Therefore, from Equation (33) the series

$$
\zeta(3)=\sum_{m=1}^{\infty} \frac{W\left(q_{m-1}^{(i, j)}, p_{m-1}^{(i, j)}\right)}{q_{m-1}^{(i, j)} q_{m}^{(i, j)}},
$$

converges, which leads to different series representations of this number for different sequences $\left(q_{n}^{(i, j)}\right)_{n \geq 0}$.

Example 1. Let $i=1$ and $j=2$. From Equation (29) we see that

$$
W\left(q_{n}^{(1,2)}, p_{n}^{(1,2)}\right)=\frac{2 \mathcal{N}_{n, \vartheta}}{n^{3}(n+1)^{3}}, \quad n \geq 1,
$$

where

$$
\mathcal{N}_{n, \vartheta}=24 \vartheta^{2} n^{3}+30 \vartheta^{2} n^{2}+16 \vartheta^{2} n+3 \vartheta^{2}+9 \vartheta n^{2}+5 \vartheta n+\vartheta-12 n^{3}-21 n^{2}-11 n-2, \quad \vartheta \in \mathbb{N} \backslash\{1\} .
$$

The right hand side of the above expression $\mathcal{N}_{n, \vartheta}$ is a polynomial of degree 2 in $\vartheta$, whose zeros $\vartheta_{1, n}$ and $\vartheta_{2, n}$ are non-integers depending on $n$. Therefore, $\mathcal{N}_{n, \vartheta} \neq 0$ for $\vartheta \in \mathbb{N} \backslash\{1\}$, and $n \geq 1$. Indeed,

$$
\vartheta_{k, n}=\frac{-\left(1+5 n+9 n^{2}\right) \pm \sqrt{25+270 n+1239 n^{2}+3090 n^{3}+4425 n^{4}+3456 n^{5}+1152 n^{6}}}{2\left(3+16 n+30 n^{2}+24 n^{3}\right)}, \quad k=1,2,
$$

and $\lim _{n \rightarrow \infty} \vartheta_{1, n}=1 / \sqrt{2}$ and $\lim _{n \rightarrow \infty} \vartheta_{2, n}=-1 / \sqrt{2}$. Finally, from (35)

$$
\zeta(3)=\sum_{n=1}^{\infty} \frac{2 \mathcal{N}_{n, \vartheta}}{n^{3}(n+1)^{3} q_{n}^{(1,2)} q_{n+1}^{(1,2)}}+\frac{p_{1}^{(1,2)}}{2(3 \vartheta-2)},
$$

where $q_{n}^{(1,2)}=2\left(\sum_{k=0}^{n}\left(\begin{array}{c}n+k \\ k\end{array}\right)^{2}\left(\begin{array}{c}n \\ k\end{array}\right)^{2} \frac{(\vartheta n-k)}{n+k}\right), n \geq 1$.

Theorem 1. The sequences $\left(p_{n}^{(i, j)}\right)_{n \geq 1^{\prime}}\left(q_{n}^{(i, j)}\right)_{n \geq 1^{\prime}}$ and $\left(r_{n}^{(i, j)}\right)_{n \geq 1}$ satisfy the following second order holonomic difference equation

$$
\alpha_{n, \Omega} y_{n+2}+\beta_{n, \Omega} y_{n+1}+\gamma_{n, \Omega} y_{n}=0, \quad n=1,2, \ldots,
$$


where

$$
\alpha_{n, \Omega}=W_{n}^{(i, j)} \Theta_{n}, \quad \gamma_{n, \Omega}=W_{n+1}^{(i, j)} \Theta_{n}, \quad \Theta_{n} \in \mathbb{Z},
$$

and $\beta_{n, \Omega}$ is a polynomial of degree $p \in \mathbb{N}$ in the variable $n$. The initial values $y_{1}$ and $y_{2}$ are given in (27) and (28).

Proof. From Lemma 1, we have

$$
\begin{aligned}
& r_{n+2}^{(i, j)}=\frac{q_{n+2}^{(i, j)}}{q_{n+1}^{(i, j)}} r_{n+1}^{(i, j)}-\frac{W_{n+1}^{(i, j)}}{q_{n+1}^{(i, j)}}, \\
& r_{n}^{(i, j)}=\frac{q_{n}^{(i, j)}}{q_{n+1}^{(i, j)}} r_{n+1}^{(i, j)}+\frac{W_{n}^{(i, j)}}{q_{n+1}^{(i, j)}},
\end{aligned}
$$

where $W_{n}^{(i, j)}=W\left(q_{n}^{(i, j)}, p_{n}^{(i, j)}\right)$. Thus, multiplying the first equation by $W_{n}^{(i, j)}$, the second one by $-W_{n+1}^{(i, j)}$, and adding, one gets

$$
W_{n}^{(i, j)} r_{n+2}^{(i, j)}-\left(W_{n}^{(i, j)} \frac{q_{n+2}^{(i, j)}}{q_{n+1}^{(i, j)}}+W_{n+1}^{(i, j)} \frac{q_{n}^{(i, j)}}{q_{n+1}^{(i, j)}}\right) r_{n+1}^{(i, j)}+W_{n+1}^{(i, j)} r_{n}^{(i, j)}=0, \quad n \geq 1 .
$$

Multiplying this equation by an integer constant $\Theta_{n}$, we have

$$
\alpha_{n, \Omega} r_{n+2}^{(i, j)}-\tilde{\beta}_{n, \Omega} r_{n+1}^{(i, j)}+\gamma_{n, \Omega} r_{n}^{(i, j)}=0, \quad n=1,2, \ldots
$$

where $\alpha_{n, \Omega}=W_{n}^{(i, j)} \Theta_{n}$, and $\gamma_{n, \Omega}=W_{n+1}^{(i, j)} \Theta_{n}$. Since for $n=1,2, \ldots, W_{n}^{(i, j)}, W_{n+1}^{(i, j)} \in \mathbb{Q}$, there exists $\Theta_{n} \in \mathbb{Z}$ such that $\alpha_{n, \Omega}, \gamma_{n, \Omega} \in \mathbb{Q}$. Moreover,

$$
\tilde{\beta}_{n, \Omega}=\alpha_{n, \Omega} \frac{q_{n+2}^{(i, j)}}{q_{n+1}^{(i, j)}}+\gamma_{n, \Omega} \frac{q_{n}^{(i, j)}}{q_{n+1}^{(i, j)}} .
$$

This leads to the second order recurrence relation

$$
\alpha_{n, \Omega} y_{n+2}-\tilde{\beta}_{n, \Omega} y_{n+1}+\gamma_{n, \Omega} y_{n}=0, \quad n=1,2, \ldots,
$$

satisfied by the sequences $\left(q_{n}^{(i, j)}\right)_{n \geq 1},\left(p_{n}^{(i, j)}\right)_{n \geq 1}$ and $\left(r_{n}^{(i, j)}\right)_{n \geq 1}$. Furthermore, from (40) one can write

$$
\tilde{\beta}_{n, \Omega}=\alpha_{n, \Omega} \frac{p_{n+2}^{(i, j)}}{p_{n+1}^{(i, j)}}+\gamma_{n, \Omega} \frac{p_{n}^{(i, j)}}{p_{n+1}^{(i, j)}}=\alpha_{n, \Omega} \frac{r_{n+2}^{(i, j)}}{r_{n+1}^{(i, j)}}+\gamma_{n, \Omega} \frac{r_{n}^{(i, j)}}{r_{n+1}^{(i, j)}}=\alpha_{n, \Omega} \frac{q_{n+2}^{(i, j)}}{q_{n+1}^{(i, j)}}+\gamma_{n, \Omega} \frac{q_{n}^{(i, j)}}{q_{n+1}^{(i, j)}} .
$$

From (39) we see that there exists a positive integer $p$ such that the sequence $\tilde{\beta}_{n, \Omega} / n^{p}$ converges when $n \rightarrow \infty$. Thus, by setting $n=1, \ldots, p+1$ in relation (39) we obtain a linear system of equations for determining explicitly the coefficients of $\tilde{\beta}_{n}=a_{p} n^{p}+a_{p-1} n^{p-1}+\cdots+a_{0}$. The solution of the resulting system gives us the coefficient $\beta_{n, \Omega}$ given in (36) and (37), where $\tilde{\beta}_{n, \Omega}=-\beta_{n, \Omega}$. This completes the proof of the theorem.

Example 2. Consider the sequences $\left(p_{n}^{(1,2)}\right)_{n \geq 1}\left(q_{n}^{(1,2)}\right)_{n \geq 1}$ and $\left(r_{n}^{(1,2)}\right)_{n \geq 1}$. We show that the second order recurrence relation

$$
\alpha_{n, \vartheta} y_{n+2}+\beta_{n, \vartheta} y_{n+1}+\gamma_{n, \vartheta} y_{n}=0, \quad n=1,2, \ldots, \quad \vartheta \in \mathbb{N} \backslash\{1\},
$$


holds, where

$$
\begin{gathered}
\alpha_{n, \vartheta}=(n+2)^{3}\left(24 \vartheta^{2} n^{3}+30 \vartheta^{2} n^{2}+16 \vartheta^{2} n+3 \vartheta^{2}+9 \vartheta n^{2}+5 \vartheta n+\vartheta\right. \\
\left.-12 n^{3}-21 n^{2}-11 n-2\right), \\
\beta_{n, \vartheta}=-2\left(408 \vartheta^{2} n^{6}+2346 \vartheta^{2} n^{5}+5336 \vartheta^{2} n^{4}+6130 \vartheta^{2} n^{3}+3810 \vartheta^{2} n^{2}\right. \\
+1268 \vartheta^{2} n+172 \vartheta^{2}+153 \vartheta n^{5}+769 \vartheta n^{4}+1417 \vartheta n^{3}+1143 \vartheta n^{2}+382 \vartheta n \\
\left.+52 \vartheta-204 n^{6}-1275 n^{5}-3181 n^{4}-4011 n^{3}-2667 n^{2}-886 n-120\right), \\
\gamma_{n, \vartheta}=n^{3}\left(24 \vartheta^{2} n^{3}+102 \vartheta^{2} n^{2}+148 \vartheta^{2} n+73 \vartheta^{2}+9 \vartheta n^{2}+23 \vartheta n+15 \vartheta\right. \\
\left.-12 n^{3}-57 n^{2}-89 n-46\right),
\end{gathered}
$$

and the initial values $y_{1}$ and $y_{2}$ are given in (27) and (28).

Let

$$
W_{n}=W_{n}^{(1,2)}=\frac{2 \mathcal{N}_{n}}{n^{3}(n+1)^{3}}
$$

Then, from (38), setting $\Theta_{n}=n^{3}(n+1)^{3}(n+2)^{3}$, we have

$$
\alpha_{n, \vartheta} r_{n+2}^{(1,2)}-\tilde{\beta}_{n, \vartheta} r_{n+1}^{(1,2)}+\gamma_{n, \vartheta} r_{n}^{(1,2)}=0, \quad n=1,2, \ldots,
$$

where $\alpha_{n, \vartheta}$ and $\gamma_{n, \vartheta}$ are given in (42), and

$$
\tilde{\beta}_{n, \vartheta}=\alpha_{n, \vartheta} \frac{q_{n+2}^{(1,2)}}{q_{n+1}^{(1,2)}}+\gamma_{n, \vartheta} \frac{q_{n}^{(1,2)}}{q_{n+1}^{(1,2)}}
$$

Denoting $\tilde{\beta}_{n}=a n^{6}+b n^{5}+c n^{4}+d n^{3}+e n^{2}+f n+g$ and setting $n=1,2, \ldots, 7$ in relation (43) we obtain a linear system of equations for determining its coefficients explicitly. Finally, one obtains the coefficient $\beta_{n}$ given in (42).

Observe that the characteristic equation for the above equation with coefficients (42) is $t^{2}-34 t+1$, which coincides with the one derived from (1). Using Poincaré's Theorem [6] the asymptotic estimates $q_{n}^{(1,2)}=$ $\mathcal{O}\left((\sqrt{2}+1)^{4 n}\right)$ and $r_{n}^{(1,2)}=\mathcal{O}\left((\sqrt{2}-1)^{4 n}\right)$ hold. In Section 4 a more accurate asymptotic expression for general cases, when $i=1,2,3,4$, and $j=1,2,3$, are studied.

An interesting consequence of Theorem 1 is the continued fraction representation of the number $\zeta(3)$. Below we present one of a number of possible continued fraction representations that follows from our results.

Example 3. Let $\vartheta=2$. The following continued fraction expansion holds:

$$
\zeta(3)=\frac{9 \mid}{\mid 8}+\frac{-184 \mid}{\mid 359}+\frac{-30672 \mid}{\mid \mathcal{Q}_{3}}+\frac{\mathcal{P}_{4} \mid}{\mid \mathcal{Q}_{4}}+\cdots+\frac{\mathcal{P}_{n} \mid}{\mid \mathcal{Q}_{n}}+\cdots,
$$

where

$$
\mathcal{P}_{n}=-9(n-2)^{3}(n-1)^{3}\left(28 n^{3}-213 n^{2}+543 n-464\right)\left(28 n^{3}-45 n^{2}+27 n-6\right),
$$

and

$$
\mathcal{Q}_{n}=6\left(476 n^{6}-2907 n^{5}+7077 n^{4}-8715 n^{3}+5715 n^{2}-1926 n+264\right) .
$$

Recall that two continued fractions

$$
a_{0}+\frac{b_{1} \mid}{\mid a_{1}}+\frac{b_{2} \mid}{\mid a_{2}}+\frac{b_{3} \mid}{\mid a_{3}}+\cdots+\frac{b_{n} \mid}{\mid a_{n}}, \quad a_{0}^{\prime}+\frac{b_{1}^{\prime} \mid}{\mid a_{1}^{\prime}}+\frac{b_{2}^{\prime} \mid}{\mid a_{2}^{\prime}}+\frac{b_{3}^{\prime} \mid}{\mid a_{3}^{\prime}}+\cdots+\frac{b_{n}^{\prime} \mid}{\mid a_{n}^{\prime}}
$$


are said to be equivalent if there exists a non-zero sequence $\left(c_{n}\right)_{n \geq 0}$, with $c_{0}=1$, such that (see [14], p. 20)

$$
a_{n}^{\prime}=c_{n} a_{n}, \quad n=0,1,2, \ldots, \quad b_{n}^{\prime}=c_{n} c_{n-1} b_{n}, \quad n=1,2, \ldots
$$

Furthermore, if $\left(p_{n}\right)_{n \geq-1}$ and $\left(q_{n}\right)_{n \geq-1}$ are two sequences such that $q_{-1}=0, p_{-1}=q_{0}=1$ and the Casorati determinant $p_{n} q_{n-1}-p_{n-1} q_{n}$ is different from zero for $n=0,1,2, \ldots$, then there exists a unique continued fraction

$$
a_{0}+\frac{b_{1} \mid}{\mid a_{1}}+\frac{b_{2} \mid}{\mid a_{2}}+\frac{b_{3} \mid}{\mid a_{3}}+\cdots+\frac{b_{n} \mid}{\mid a_{n}}
$$

whose $n$-th numerator is $p_{n}$ and $n$-th denominator is $q_{n}$, for each $n \geq 0$. More precisely (see [14, $p$. 31])

$$
\begin{gathered}
a_{0}=p_{0}, \quad a_{1}=q_{1}, \quad b_{1}=p_{1}-p_{0} q_{1} \\
a_{n}=\frac{p_{n} q_{n-2}-p_{n-2} q_{n}}{p_{n-1} q_{n-2}-p_{n-2} q_{n-1}}, \quad b_{n}=\frac{p_{n-1} q_{n}-p_{n} q_{n-1}}{p_{n-1} q_{n-2}-p_{n-2} q_{n-1}}, \quad n=2,3, \ldots
\end{gathered}
$$

Now, by setting $p_{-1}=q_{0}=1, p_{0}=q_{-1}=0$ in the case $\vartheta=2$, one gets

$$
a_{1}=8, \quad a_{2}=359 / 24, \quad b_{1}=9, \quad b_{2}=-23 / 3 \text {. }
$$

Moreover, from the recurrence relation (41), one has

$$
y_{n}=-\frac{\beta_{n-2}}{\alpha_{n-2}} y_{n-1}-\frac{\gamma_{n-2}}{\alpha_{n-2}} y_{n-2}
$$

Thus, we have constructed the elements of the continued fraction (45) which satisfy (46); i.e.,

$$
a_{n}=-\frac{\beta_{n-2}}{\alpha_{n-2}}, \quad b_{n}=-\frac{\gamma_{n-2}}{\alpha_{n-2}}, \quad n \geq 3 .
$$

With the choice $c_{0}=c_{1}=1, c_{2}=24$, and $c_{n}=-\alpha_{n-2}$, for $n \geq 3$, we obtain the continued fraction (44).

\section{Asymptotic Expression for the Remainder}

In this section we use the steepest descent method to asymptotically estimate the holonomic remainder sequences $r_{n}^{(i, j)}$.

Lemma 2. The following relation holds:

$$
\frac{1}{2 \pi \mathrm{i}} \int_{-1 / 2-\mathrm{i} \infty}^{-1 / 2+\mathrm{i} \infty} R_{1, n}^{(i, j)}(v)\left(\frac{\pi}{\sin \pi v}\right)^{2} d v=\sum_{t=0}^{\infty} R_{2, n}^{(i, j)}(t) .
$$

Proof. The integral (47) can be expressed as a limit of contour integrals along the contour $\Gamma_{n, i, j}$ that runs along the imaginary line from $-1 / 2+\mathrm{i} L_{n, i, j}$ to $-1 / 2-\mathrm{i} L_{n, i, j}$ and then counterclockwise along a semicircle centered at $-1 / 2$ from $-1 / 2+\mathrm{i} L_{n, i, j}$ to $-1 / 2-\mathrm{i} L_{n, i, j}$. The semicircle radius $L_{n, i, j}>n+2$ guarantees that $n+1$ singularities of the integrand function are enclosed within the curve. The rational function $R_{1, n}^{(i, j)}(t)=\mathcal{O}\left(L_{n, i, j}^{-2}\right)$ on the arc of $\Gamma_{n, i, j}$, while the function $(\sin \pi z)^{-1}$ is bounded. Using the residue theorem, one can then compute (47). Indeed,

$$
\operatorname{Res}_{t=n}\left(R_{1, n}^{(i, j)}(z)\left(\frac{\pi}{\sin \pi z}\right)^{2}\right)=R_{2, n}^{(i, j)}(n), \quad n=0,1,2, \ldots
$$


which can be easily checked by considering the following expansions at the integers

$$
\begin{aligned}
R_{1, n}^{(i, j)}(z) & =R_{1, n}^{(i, j)}(n)+R_{2, n}^{(i, j)}(n)(z-n)+\mathcal{O}\left((z-n)^{2}\right), \\
\left(\frac{\pi}{\sin \pi z}\right)^{2} & =\frac{1}{(z-n)^{2}}+\mathcal{O}(1) .
\end{aligned}
$$

Therefore,

$$
\frac{1}{2 \pi \mathrm{i}} \int_{-1 / 2-\mathrm{i} \infty}^{-1 / 2+\mathrm{i} \infty} R_{1, n}^{(i, j)}(v)\left(\frac{\pi}{\sin \pi v}\right)^{2} d v=\sum_{t=0}^{\infty} R_{2, n}^{(i, j)}(t),
$$

holds.

Theorem 2. The following asymptotic formula holds:

$$
r_{n}^{(i, j)}=\frac{-\pi^{3 / 2} \eta^{(i, j)}}{n^{3 / 2-\delta_{i, 4} 2^{1 / 4}}}(\sqrt{2}-1)^{4 n}(1+o(1)), \text { where }\left(\eta^{(i, j)}\right)_{4,3}=\left(\begin{array}{rrr}
-1 & -\frac{|\vartheta|}{\vartheta} & 1 \\
1 & \frac{|\vartheta|}{\vartheta} & 1 \\
1 & \frac{|\vartheta|}{\vartheta} & -1 \\
1 & 1 & -1
\end{array}\right) .
$$

Proof. From relations (20)-(24), we have

$$
\begin{aligned}
q_{n}^{(i, j)} \zeta(3)-p_{n}^{(i, j)} & =2 \sum_{k=0}^{n-\delta_{i, 3}} b_{k, n-\delta_{i, 3}}^{(i, j)} \sum_{l=1}^{\infty} \frac{1}{l^{3}}-2 \sum_{k=1}^{n-\delta_{i, 3}} b_{k, n-\delta_{i, 3}}^{(i, j)} \sum_{l=1}^{k} \frac{1}{l^{3}}+\sum_{k=1}^{n} a_{k, n}^{(i, j)} \sum_{l=1}^{k} \frac{1}{l^{2}} \\
& =\sum_{t=0}^{\infty}\left(\sum_{k=0}^{n-\delta_{i, 3}} \frac{2 b_{k, n-\delta_{i, 3}}^{(i, j)}}{(t+k+1)^{3}}-\sum_{k=0}^{n} \frac{a_{k, n}^{(i, j)}}{(t+k+1)^{2}}\right)=\sum_{t=0}^{\infty} R_{2, n}^{(i, j)}(t)=r_{n}^{(i, j)},
\end{aligned}
$$

which, combined with expression (47), yields

$$
r_{n}^{(i, j)}=\frac{1}{2 \pi \mathrm{i}} \int_{1 / 2-\mathrm{i} \infty}^{1 / 2+\mathrm{i} \infty} R_{1, n}(v-1)\left(\frac{\pi}{\sin \pi v}\right)^{2} \theta^{(i, j)}(v-1) d v .
$$

Taking into account that

$$
\log \theta^{(i, j)}(v-1)= \begin{cases}\log \frac{(v+n)^{\delta_{i, 3}}(v+n+\rho-1)^{\delta_{i, 2}+1}}{(v-n)^{2-\delta_{i, 1}}}, & j=1, \\ \log \frac{(v+n)^{\delta_{i, 3}}(v+n \vartheta)^{\delta_{i, 2}+1}}{(v-n)^{2-\delta_{i, 1}}}, & j=2, \\ \log \frac{(v+n)^{\delta_{i, 3}}(v(v-1)-\chi n-\psi)^{\delta_{i, 2}+1}}{(v-n)^{2-\delta_{i, 1}}}, & j=3,\end{cases}
$$

a straightforward computation shows that for $v=(n+1) t$

$$
\begin{aligned}
\log \frac{(1-v)_{n}^{2}}{(v)_{n+1}^{2}}\left(\frac{\pi}{\sin \pi v}\right)^{2} \theta^{(i, j)}(v-1)=\log g^{(i, j)}(t) & +2(n+1) f(t) \\
& -\left(2+\delta_{i, 4}\right) \log (n+1)+2 \log 2 \pi+\mathcal{O}\left(n^{-1}\right),
\end{aligned}
$$


where

$$
g^{(i, j)}(t)= \begin{cases}\frac{(t+1)^{2-\delta_{i, 1}-\delta_{i, 4}}}{(t-1)^{2-\delta_{i, 1}}} g(t), & j=1 \\ \frac{(t+1)^{\delta_{i, 3}}(t+\vartheta)^{\delta_{i, 2}+1}}{(t-1)^{2-\delta_{i, 1}}} g(t), & j=2 \\ \frac{(t+1)^{\delta_{i, 3}}(v t-\chi)^{\delta_{i, 2}+1}}{(t-1)^{2-\delta_{i, 1}}} g(t), & j=3\end{cases}
$$

and

$$
g(t)=\frac{1+t}{(1-t) t^{2}}, \quad f(t)=(1-t) \log (1-t)+2 t \log t-(1+t) \log (1+t) .
$$

Thus, expression (48) transforms into

$$
r_{n}^{(i, j)}=\frac{2 \pi \mathrm{i}}{n^{1+\delta_{i, 4}}} \int_{1 / \sqrt{2}-\mathrm{i} \infty}^{1 / \sqrt{2}+\mathrm{i} \infty} g^{(i, j)}(t) e^{2(n+1) f(t)}\left(1+\mathcal{O}\left(n^{-1}\right)\right) d t .
$$

The point $t=1 / \sqrt{2}$ is the unique maximum point for $\operatorname{Re} f(t)$ on the contour of integration. Using the steepest descent method we obtain

$$
r_{n}^{(i, j)}=-\frac{\pi^{3 / 2} \eta^{(i, j)}}{2^{1 / 4} n^{1+\delta_{i, 4}}(n+1)^{1 / 2}}\left|g^{(i, j)}\left(\sqrt{2^{-1}}\right)\right|(\sqrt{2}-1)^{4 n+4}\left(1+\mathcal{O}\left(n^{-1}\right)\right),
$$

which gives the required estimate.

Finally, we point out that the holonomic sequences of rational approximants obtained in this paper reprove the irrationality of $\zeta(3)$. Suppose on the contrary that $\zeta(3)=p / q$, where $p \in \mathbb{Z}, q \in \mathbb{N}$, then

$$
q n^{\omega_{i, j}} l_{n}^{3} r_{n}^{(i, j)}=n^{\omega_{i, j}} l_{n}^{3} q_{n}^{(i, j)} p-q n^{\omega_{i, j}} l_{n}^{3} p_{n}^{(i, j)},
$$

is an integer different from zero. Notice that using the inclusions (26) and the fact that $l_{n}^{s} \sum_{l=1}^{k} \frac{1}{l^{s}} \in \mathbb{Z}$, $k=0,1, \ldots, n, s \in \mathbb{Z}^{+}$, one obtains $n^{\omega_{i, j}} q_{n}^{(i, j)} \in \mathbb{Z}$ and $n^{\omega_{i, j}} l_{n}^{3} p_{n}^{(i, j)} \in \mathbb{Z}$. Therefore,

$$
1 \leq q n^{\omega_{i, j}} l_{n}^{3}\left|r_{n}^{(i, j)}\right|=\mathcal{O}\left(l_{n}^{3}(\sqrt{2}-1)^{4 n}\right),
$$

contradicting the above assumption for $\zeta(3)$, since for any $\varepsilon>0$ and any sufficiently large $n$ the estimate $l_{n}<e^{(1+\varepsilon) n}$ yields $e^{3}(\sqrt{2}-1)^{4}<1$.

\section{Discussion of Results}

We discuss our results with the aid of graphics and tables. Denote $\pi_{n}^{(i, j)}=p_{n}^{(i, j)} / q_{n}^{(i, j)}$, where the integers $i, j$ are such that $0 \leq i \leq 4,0 \leq j \leq 3$. By $\pi_{n}^{(0,0)}$ we denote the Apéry's approximants, where

$$
\begin{gathered}
q_{n}^{(0,0)}=\sum_{k=0}^{n} b_{k}^{(n)}, \quad b_{k}^{(n)}=\left(\begin{array}{c}
n+k \\
k
\end{array}\right)^{2}\left(\begin{array}{l}
n \\
k
\end{array}\right)^{2}, \\
p_{n}^{(0,0)}=\sum_{k=1}^{n}\left(b_{k}^{(n)} H_{k}^{(3)}-a_{k}^{(n)} H_{k}^{(2)}\right), \quad a_{k}^{(n)}=\left(H_{n+k}-2 H_{k}+H_{n-k}\right) b_{k}^{(n)} .
\end{gathered}
$$

In Figure 1, a comparison between twelve selected rational approximants from the set $\pi_{n}^{(i, j)}$ corresponding to different choices of parameters and Apéry's approximants $\pi_{n}^{(0,0)}$ is given. This comparison is depicted by means of a rectangular array of squares formed by thirteen rows and 
nine columns in a grayscale output, in which the color of each square is determined by the value of the function

$$
f\left(\pi_{n}^{(i, j)}\right)=\left|\left(\log \left|\zeta(3)-\pi_{n}^{(i, j)}\right|\right)^{-1}\right|, \quad 0 \leq i \leq 4,0 \leq j \leq 3, \quad n=2, \ldots 10,
$$

ranges from $0.0144346 \ldots$ to $0.137009 \ldots$ The values close to the minimum of (50) are shown as white squares while its maxima are shown as dark squares. Indeed, ten iterations (see columns in Figure 1) are enough to show the accuracy of our results in the approximation to $\zeta(3)$. Clearly, in Figure 1 the darkness decreases as the number of iterations grows, which is in accordance with the analytical results on the asymptotic behavior of the remainders discussed in Section 4. In addition, in Tables 1 and 2, we compare the rates of convergence of four selected cases among infinitely many options of the studied holonomic rational approximants versus $\pi_{n}^{(0,0)}$; namely, $\pi_{n}^{(1,1)}$ for $\rho=2, \pi_{n}^{(1,2)}$ for $\vartheta=2$, and $\pi_{n}^{(1,3)}$ for $v=\chi=\psi=1$.

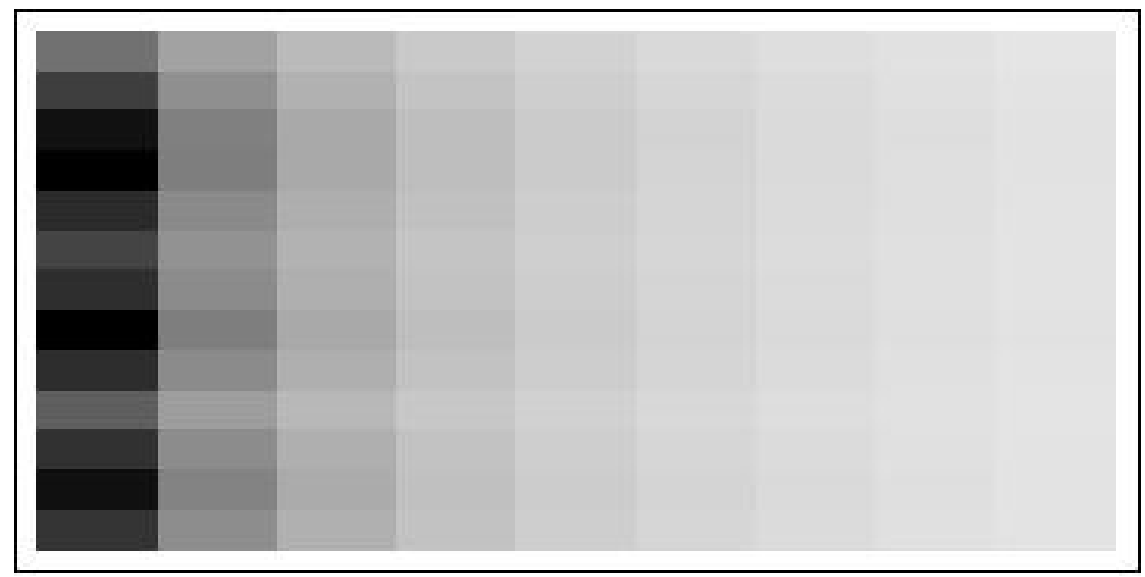

Figure 1. From left to right are displayed, in gray-scale output, the values of function (50) for $n=$ $2, \ldots, 10$, and from top to bottom its arguments are: $\pi_{n}^{(0,0)}, \pi_{n}^{(1,1)}$ for $\rho=2, \pi_{n}^{(2,1)}$ for $\rho=4, \pi_{n}^{(3,1)}$ for $\rho=913, \pi_{n}^{(4,1)}$ for $\rho=23, \pi_{n}^{(1,2)}$ for $\vartheta=2, \pi_{n}^{(2,2)}$ for $\vartheta=784, \pi_{n}^{(3,2)}$ for $\vartheta=93, \pi_{n}^{(4,2)}$ for $\vartheta=57, \pi_{n}^{(1,3)}$ for $v=\chi=\psi=1, \pi_{n}^{(2,3)}$ for $v=49, \chi=891, \psi=97, \pi_{n}^{(3,3)}$ for $v=413, \chi=732, \psi=231$, and $\pi_{n}^{(4,3)}$ for $v=713, \chi=3427, \psi=231$. For $n=10$ (last column), a high level of coincidence between all rational approximants was already observed .

Table 1. Comparison between Apéry's rational approximants $\pi_{n}^{(0,0)}$ and holonomic rational approximants $\pi_{n}^{(1,1)}$ for $\rho=2$.

\begin{tabular}{ccccc}
\hline$n$ & $\pi_{n}^{(0,0)}$ & $\zeta(3)-\pi_{n}^{(0,0)}$ & $\pi_{n}^{(1,1)}$ & $\zeta(3)-\pi_{n}^{(1,1)}$ \\
\hline 2 & $\frac{351}{292}$ & $2.109 \times 10^{-6}$ & $\frac{1327}{1104}$ & 0.00006 \\
\hline 3 & $\frac{62,531}{52,020}$ & $1.968 \times 10^{-9}$ & $\frac{104,377}{86,832}$ & $5.776 \times 10^{-8}$ \\
\hline 4 & $\frac{11,444,695}{9,504,288}$ & $1.778 \times 10^{-12}$ & $\frac{58,642,219}{48,769,920}$ & $5.211 \times 10^{-11}$ \\
\hline$\vdots$ & $\vdots$ & $\vdots$ & $\vdots$ & $\vdots$ \\
\hline 50 & $\cdot$ & $2.795 \times 10^{-153}$ & $\cdot$ & $9.250 \times 10^{-152}$ \\
\hline
\end{tabular}


Table 2. Comparison between Apéry's rational approximants $\pi_{n}^{(0,0)}$ and holonomic rational approximants $\pi_{n}^{(1,2)}$ for $\vartheta=2$, and $\pi_{n}^{(1,3)}$ for $v=\chi=\psi=1$.

\begin{tabular}{ccccccc}
\hline$n$ & $\pi_{n}^{(\mathbf{0 , 0})}$ & $\zeta(3)-\pi_{n}^{(\mathbf{0 , 0})}$ & $\pi_{n}^{(\mathbf{1 , 2})}$ & $\zeta(3)-\pi_{n}^{(\mathbf{1 , 2})}$ & $\pi_{n}^{(\mathbf{1 , 3})}$ & $\zeta(3)-\pi_{n}^{(\mathbf{1 , 3})}$ \\
\hline 2 & $\frac{351}{292}$ & $2.109 \times 10^{-6}$ & $\frac{1077}{896}$ & 0.00004 & $\frac{2231}{1856}$ & $9.489 \times 10^{-6}$ \\
\hline 3 & $\frac{62,531}{52,020}$ & $1.968 \times 10^{-9}$ & $\frac{1987}{1653}$ & $3.686 \times 10^{-8}$ & $\frac{783,217}{651,564}$ & $6.216 \times 10^{-9}$ \\
\hline 4 & $\frac{11,424,695}{9,504,288}$ & $1.778 \times 10^{-12}$ & $\frac{34,774,333}{28,929,024}$ & $3.006 \times 10^{-11}$ & $\frac{118,221,931}{98,349,696}$ & $4.550 \times 10^{-12}$ \\
\hline$\vdots$ & $\vdots$ & $\vdots$ & $\vdots$ & $\vdots$ & $\vdots$ & $\vdots$ \\
\hline 50 & $\cdot$ & $2.795 \times 10^{-153}$ & $\cdot$ & $3.505 \times 10^{-152}$ & $\cdot$ & $3.114 \times 10^{-153}$ \\
\hline
\end{tabular}

In the above discussions the computational software [15] was used.

\section{Concluding Remarks}

The main point of interest in this paper is the deduction in Section 3 of the holonomic difference Equation (36). The non-holonomic equation (Equation (1)) was the cornerstone of Apéry's original proof [1], which seemed unrelated to constructive approximation. The holonomicity is a consequence of the Hermite-Padé approximation problems formulated near infinity (5) and (6) along with some extra conditions for the vector polynomials involved. In the present contribution, we used a straightforward technique involving Casorati determinants to obtain the recurrence coefficients in the holonomic difference equation. A set of holonomic rational approximants with good rates of convergence to $\zeta(3)$ is discussed in Sections 4 and 5. In Section 4, most of the used techniques are standard in the field, but we present them for completeness of the study; in particular, the use of the steepest descent method.

In the study of rational approximants for the Euler constant [16], the authors also used Casorati determinants to reduced 10-term recurrence relations (which involved Hermite-Padé approximants) to the 4-term recurrences.

Other techniques for deriving recurrence relations rely on the algorithm of creative telescoping. The use of these techniques is more complicated in our context due to the dependence of integer parameters (up to three) in addition to the non-holonomic variable $n$. In the literature, several results connect recurrence relations, continued fractions, and irrationality as well as the use of contiguous relations of hypergeometric series to derive rational approximants; however, similar results involving a holonomic phenomenon are absent. This paper focuses on the study of such a situation in the well known case of $\zeta$ (3) (an early draft of our work can be found in [17], also contained in the Ph. D. Thesis of the second author).

The other approach to the irrationality of $\zeta(3)$ deals with the perturbation of the rational function $R_{1, n}(t)=\frac{(-t)_{n}^{2}}{(t+1)_{n+1}^{2}}, n \in \mathbb{N}$, introduced by Beukers in ([18], p. 97) and by Nesterenko in $[19,20]$. In the next example, for a couple of rational perturbations of $R_{1, n}(t)$, the generated sequences of rational approximants differ from the Apéry's approximants given in (49). Nevertheless, such sequences (as will be discussed) might not guarantee a priori that reproving the irrationality of $\zeta(3)$ is possible. Alternatively, we propose deriving the perturbed rational functions (as in expression (22)) from a Hermite-Padé approximation problem along with extra conditions.

Consider the following two perturbations:

$$
R_{1, n}^{(1)}=\frac{(-t)_{n}^{2}}{(t+1)_{n+1}^{2}}\left(\frac{t+n+1}{t+n+2}\right)
$$

and

$$
R_{1, n}^{(2)}=\frac{(-t)_{n}^{2}}{(t+1)_{n+1}^{2}}\left(\frac{a_{n} t^{2}+b_{n}}{t-n+1}\right)
$$


where $a_{n}=4 n\left(2 H_{n}-H_{2 n-1}\right)-1, b_{n}=(n+1) a_{n}-2 n$. For (51) and (52), the derived corresponding sequences $\left(p_{n}^{(i)}\right)_{n \geq 0}$ and $\left(q_{n}^{(i)}\right)_{n \geq 0^{\prime}}, i=1,2$, fail to prove the irrationality of $\zeta(3)$ because: (1) The multiplication by $l_{n}^{3}$ in the resulting linear form $q_{n}^{(i)} \zeta(3)-p_{n}^{(i)}=r_{n}^{(i)}$ will not eliminate the rational terms in the sequences $l_{n}^{3}\left(p_{n}^{(i)}\right)_{n \geq 0}$. (2) The additional requirements to transform them into integers cause the error-term sequence to diverge. However, the convergence of $\left(p_{n}^{(i)} / q_{n}^{(i)}\right)_{n \geq 0}$ to the number $\zeta(3)$ is good, as it is depicted in Figure 2, and their rates of convergence are as reasonable as Apéry's approximants.

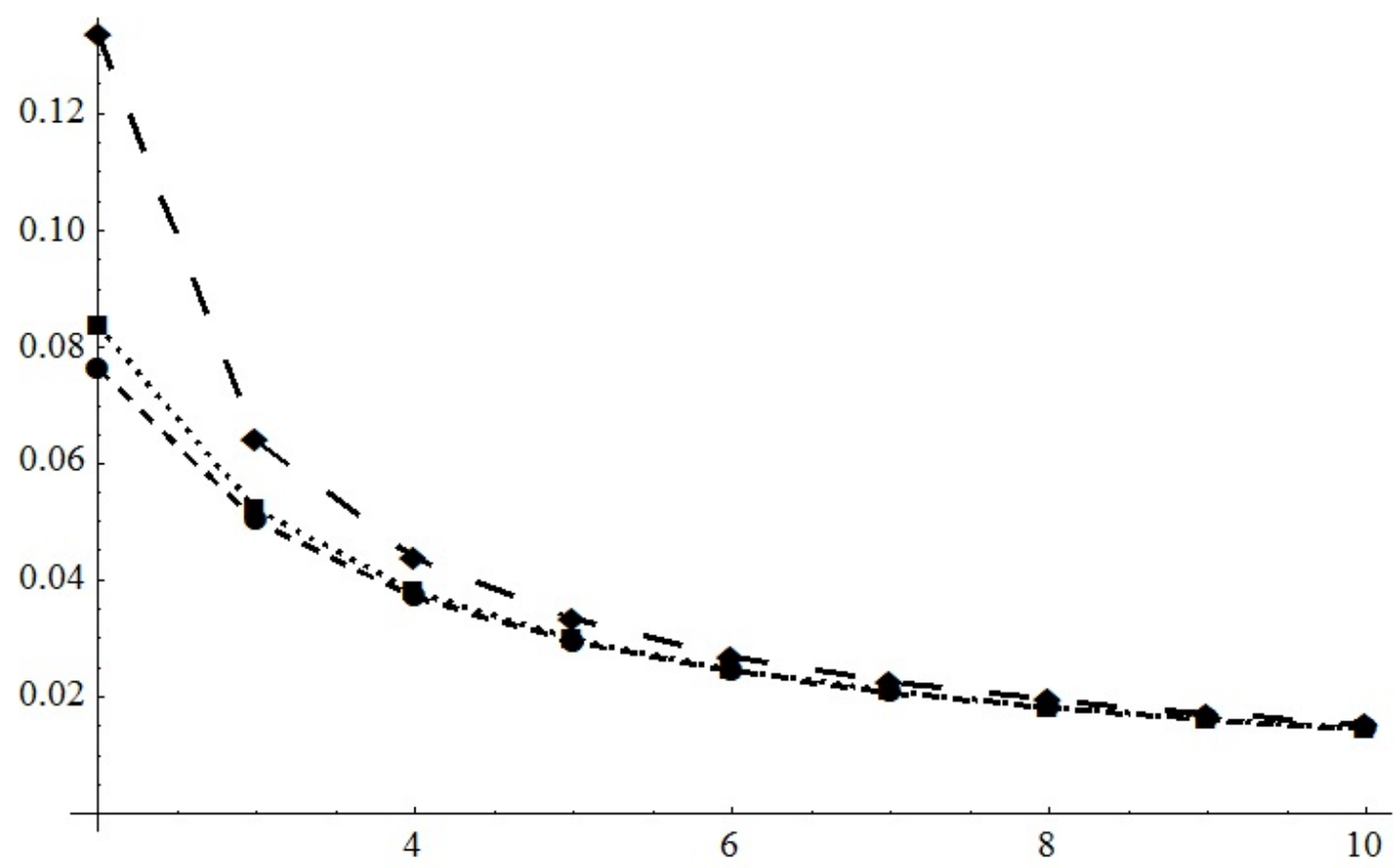

Figure 2. Function (50) is plotted for $n=2, \ldots, 10$ in the following three situation: Symbol $\bullet$ is used for depicting Apéry's approximants, while and are used for the approximants derived from (51) and (52), respectively.

Author Contributions: Conceptualization, J.A. and A.S.-L.; methodology, J.A.; software, A.S.-L.; validation, A.S.-L.; formal analysis, J.A.; investigation, J.A. and A.S.-L.; resources, J.A. and A.S.-L.; data curation, A.S.-L.; writing - original draft preparation, J.A. and A.S.-L.; writing-review and editing, J.A.; visualization, J.A. and A.S.-L.; supervision, J.A.; project administration, J.A.; funding acquisition, J.A.

Funding: The research of J.A. was funded by Agencia Estatal de Investigación of Spain, grant number PGC-2018-096504-B-C33 and Comunidad Autónoma de Madrid, grant number CC-G08-UC3M/ESP-4516.

Conflicts of Interest: The authors declare no conflict of interest

\section{Reference}

1. Apéry, R. Irrationalité de $\zeta(2)$ et $\zeta(3)$. Astérisque 1979, 61, 11-13.

2. Sloane, N.J.A. The On-Line Encyclopedia of Integer Sequences; OEIS Foundation Inc.: Highland Park, NJ, USA, 2019.

3. der Poorten, A.V. A proof that Euler missed... Apéry's proof of the irrationality of $\zeta(3)$. Math. Intell. 1979, 1, 195-203. [CrossRef]

4. Perron, O. Über einen Satz des Herrn Poincaré. J. Reine Angew. Math. 1909, 136, 17-37. [CrossRef]

5. Perron, O. Über die Poincarésche lineare Differenzengleichung. J. Reine Angew. Math. 1910, 137, 6-64. [CrossRef]

6. Poincaré, H. Sur les équations linéaires aux différentielles et aux différences finies. Amer. J. Math. 1885, 7, 203-258. [CrossRef] 
7. Sorokin, V.N. Hermite-Padé approximations for Nikishin systems and the irrationality of $\zeta(3)$. Commun. Mosc. Math. Soc. 1993, 49, 176-177.

8. Aptekarev, A.I. Multiple orthogonal polynomials. J. Comput. Appl. Math. 1998, 99, 423-447. [CrossRef]

9. Nikishin, E.M. On simultaneous Padé approximations. Mat. Sb. 1980, 155, 499-519. Available online: http:// www.mathnet.ru/php/archive.phtml?wshow=paper\&jrnid=sm\&paperid=2815\&option_lang=eng (accessed on 28 October 2019).

10. Nikishin, E.M.; Sorokin, V.N. Rational Approximations and Orthogonality. American Mathematics Society: Providence, RI, USA, 1991. Available online: https:/ / bookstore.ams.org/mmono-92 (accessed on 24 October 2019).

11. Álvarez-Nodarse, R.; Arvesú, J.; Marcellán, F. On the Krall-type polynomials. J. Appl. Math. 2004, 5 , 359-369. [CrossRef]

12. Due nas, H.; Marcellán, F. The Laguerre-Sobolev-Type Orthogonal Polynomials. Holonomic equation and Electrostatic interpretation. Rocky Mt. J. Math. 2011 41, 95-131. [CrossRef]

13. Zeilberger, D. The Method of Creative Telescoping. J. Symb. Comput. 1991, 11, 195-204. [CrossRef]

14. Jones, W.B.; Thron, W.J. Continued Fractions Analytic Theory and Applications (Encyclopedia of Mathematics and its Applications; Addison-Wesley: London, UK, 1980.

15. Wolfram Research, Inc. Mathematica, version 11.3; Wolfram Research, Inc.: Champaign, IL, USA, 2018.

16. Aptekarev, A.I.; Tulyakov, D.N. Four-termed recurrence relations for $\gamma$-forms. Proc. Steklov Inst. Math. 2011, 272 (Suppl. 2), 157-161. [CrossRef]

17. Arvesú, J.; Soria-Lorente, A. On new rational approximants to $\zeta(3)$. arXiv 2012, arXiv:1204.6712.

18. Beukers, F. Padé Approximations in Number Theory; Padé Approximation and Its Applications, (Amsterdam, 1980); Lecture Notes in Mathematics, Volume 888; Springer: Berlin, Germany, 1981; pp. 90-99.

19. Nesterenko, Y.V. A few remarks on $\zeta(3)$. Math. Notes 1996, 59, 625-636. [CrossRef]

20. Nesterenko, Y.V. An Elementary Proof of the Irrationality of $\zeta(3)$. Moscow Univ. Math. Bull. 2009, 64, $165-171$. [CrossRef]

(C) 2019 by the authors. Licensee MDPI, Basel, Switzerland. This article is an open access article distributed under the terms and conditions of the Creative Commons Attribution (CC BY) license (http:/ / creativecommons.org/licenses/by/4.0/). 